queries during this time. Only one patient from this group had a further colonoscopy having exhibited new symptoms. The total waiting times for all colonoscopy surveillance programmes reduced by 6 weeks. All new polypectomy referrals to the clinic were actioned within 7 days of receipt of histology.

Conclusion The development and implementation of the nurse endoscopist led virtual polyp surveillance clinic has led to a reduction in polyp and post colorectal cancer surveillance colonoscopies, prevented unnecessary procedures, reduced colon surveillance waiting times for all patients and has been widely accepted by patients, GPs and referring clinicians.

\section{P392 HOW HAS THE TRANSITION FROM FOBT TO FIT CHANGED THE BCSP COLONOSCOPY SERVICE?}

Tania Baldwin, Ajay Verma*. Kettering General Hospital NHS FT, Kettering, UK

10.1136/gutjnl-2020-bsgcampus.466

Introduction In 2019, the national bowel cancer screening programme (BCSP) switched its home stool test kit from a six windowed guaiac faecal occult blood test (FOBt), to a single sample semi-quantitive faecal immunochemical test (FIT). The FIT test is superior to $\mathrm{FOBt},{ }^{1}$ the transition to a single sample was expected to increase returns (uptake). A FIT cut-off = $120 \mu \mathrm{g} / \mathrm{g}$ was introduced so that endoscopy services weren't overwhelmed. We evaluate the impact of this switch on the colonoscopy service.

Methods Northamptonshire patients who returned a positive stool testing kit in 2019 had their data collated and analysed.

Results Of 565 patients, 356 (63.0\%) were male. 52 (9.2\%) had colorectal cancer (CRC), 308 had polyps (54.5\%). Overall; 340 (60.2\%) had CRC \&/or polyps.

Conclusion The switch from FOBt to FIT has changed the profile of patients returning a positive test. The convenience of a single sample has presumably driven an increase in returns (uptake), the $120 \mu \mathrm{g} / \mathrm{g}$ FIT test threshold has increased positivity. The age $\&$ gender profiles, and CRC detection rate, are similar. There is a higher ADR, with a significantly higher number of larger $(>10 \mathrm{~mm})$ polyps detected \& removed. In cases where polyps were identified, the mean number of adenomas removed is also significantly higher, and more likely

\begin{tabular}{llll} 
Abstract P392 Table 1 & & \\
\hline & FOBt & FIT & FIT (1st return) \\
\hline Number & 294 & 271 & 69 \\
Uptake & $62 \% *$ & $66.6 \% *$ & \\
Positivity & $1.86 \% *$ & $2.29 \% *$ & \\
Male/Female & $62.6 / 37.4 \%$ & $63.5 / 36.5 \%$ & $68.1 / 31.9 \%$ \\
Median age (yrs) & 68 & 68 & 66 \\
CRC & $26(8.8 \%)$ & $26(9.6 \%)$ & $5(7.2 \%)$ \\
Adenoma detection rate (ADR) & $154(52.4 \%)$ & $153(56.5 \%)$ & $44(63.8 \%)$ \\
$>10$ mm & $68(23.1 \%) \dagger+$ & $91(33.6 \%) \dagger$ & $26(37.7 \%) \$$ \\
$>20$ mm & $23(7.8 \%)$ & $28(10.3 \%)$ & $10(14.5 \%)$ \\
Left sided polyps & $52.2 \%$ & $58.5 \%$ & $65.6 \%$ \\
Mean adenomas removed & $1.9 \#$ & $2.3 \#$ & 2.2 \\
\hline
\end{tabular}

* Comparing Oct/Nov 2018 with Oct/Nov 2019. $\uparrow p=0.006, \pm p=0.01$

\# Number of adenomas removed in cases with polyps, $p=0.03$, be in the left side of the colon. This suggests that colonoscopists need to be prepared to perform more polypectomies, especially of larger polyps.

Interestingly, $25.5 \%$ of the FIT test positive cases were patients who had not previously returned a FOBt kit. This group were younger, and were significantly more likely to be male, have more adenomas (especially larger ones), which were more likely to be in the left colon. CRC detection was slightly lower - this may be due to those patients with cancer presenting symptomatically having not had the protection of BCSP. $^{2}$

\section{REFERENCES}

1. Moss S, Mathews C, Day TJ, et al. Increased uptake and improved outcomes of bowel cancer screening with a faecal immunochemical test: results from a pilot study within the national screening programme in England. Gut 2017;66:1631-44.

2. Logan RFA, J Patnick, C Nickerson, on behalf of the English Bowel Cancer Screening Evaluation Committee, et al. Outcomes of the Bowel Cancer Screening Programme (BCSP) in England after the first 1 million tests. Gut 2012;61:14391446.

\section{P393 CAN TELEDIETETICS IMPROVE ACCESS TO SPECIALIST DIETITIANS FOR PATIENTS WITH IBD?}

${ }^{1}$ Kapil Sahnan, ${ }^{1}$ Matthew Vincent* ${ }^{*}$ 'Lina Johansson, ${ }^{2}$ Mark Thomsit, ${ }^{2}$ Simon Heys, ${ }^{1}$ James Kinross. 'Imperial College London, London, UK; ${ }^{2}$ Make + Ship, London, UK

\subsection{6/gutjnl-2020-bsgcampus.467}

Background Specialist dietitian input, while an important part of IBD management, is often difficult for patients to access due to long wait times. Moreover, a number of dietitians are interested in part-time work, which is often not feasible in a hospital environment. We have developed a virtual dietetics platform, Mangetoo, as a way to increase flexibility for dietitians and improve patient access to IBD specialists.

Methods We performed a patient and public involvement (PPI) exercise with IBD patients sampled from Crohn's and Colitis UK to demonstrate the functionality of the platform. Following the exercise, attendants were sent a questionnaire about their views on dietetics, and whether they would be open to using the teledietetics service.

Results We received a total of 46 responses to our questionnaire. Of the patients who had previously seen a dietitian (52\%), only $20 \%$ found the interaction helpful, mostly due to seeing a generalist rather than an IBD specialist. The majority of patients (70\%) listed access to an IBD subspecialist as their most important factor in choosing a dietitian, ahead of location/access (15\%) and reputation (9\%).

$80 \%$ of respondents said they were interested in using a teledietetics service, with their main concerns being availability of a specialist dietitian, accessibility, and cost.

Conclusions Our exercise has shown that the top priority for IBD patients in engaging with dietetics was access to a subspecialist IBD dietitian. As long as a virtual dietetic platform could provide access to specialist dietitians, IBD patients would be interested in engaging with the service.

Having determined IBD patients are interested in the service in principle, we will now trial the platform, Mangetoo, using actual IBD patients and dietitians to evaluate its efficacy in replacing face-to-face dietetics consultations in practice. 